
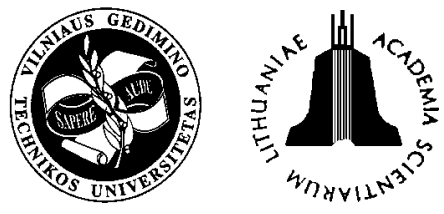

ISSN 1648-4142 print / ISSN 1648-3480 online TRANSPORT

www.transport.vtu.lt

\title{
SIMULATION-BASED FORECASTING EFFECTS OF AN ACCIDENTAL EXPLOSION ON THE ROAD. PART I: METHODOLOGICAL FRAMEWORK
}

\author{
Egidijus R. Vaidogas \\ Dept of Reinforced Concrete Structures, Vilnius Gediminas Technical University, \\ Sauletekio al.11, LT-10223 Vilnius, Lithuania.E-mail: erv@st.vtu.lt
}

Received 1 April 2006; accepted 9 May 2006

\begin{abstract}
Forecasting mechanical actions induced by accidental explosions on the road is of crucial importance to assessing potential damage to structures and non-structural property exposed to them. A logical result of such forecasting may be expressed in the form of probabilistic models. They should quantify likelihood of occurrence and physical characteristics of accidental explosions. Generally the models are to be selected under the conditions of sparse statistical information on intensities and likelihood of explosive actions. The first part of the present paper proposes a simulation-based procedure intended for selection of the probabilistic models in the absence of direct statistical data on the explosive actions. The proposed procedure is formulated in the context of the classical Bayesian approach to risk assessment. The main idea of the procedure is that statistical samples necessary for fitting the probabilistic models can be acquired from a stochastic simulation of an accident involving an explosion on the road. The proposed simulation-based procedure can be used for damage assessment and risk studies within the methodological framework provided by the above-mentioned approach. A case study illustrating an application of the proposed procedure is given in the second part of the paper.
\end{abstract}

Keywords: transportation hazardous materials, accidental action, explosion, risk, Bayesian approach, simulation.

\section{Introduction}

Man-made accidents occurring during transportation of hazardous substances by road and rail include such adverse physical phenomena as explosions, fires, and releases of dangerous materials [1-3]. Mechanical, thermal, and sometimes chemical actions (effects) induced by these phenomena can cause considerable damage to structural and non-structural property. Highly probable targets of explosions and fires are structures built in vicinity of roads. In terms of structural engineering, an action induced during a manmade accident is called an accidental action. The present paper will be focussed on one type of accidental actions, namely, actions which can be generated by an accidental explosion $\left(\mathrm{AE}^{1}\right)$ on the road.

A complete prevention of accidents capable of inducing AEs is not always possible or economically feasible. What is possible it is a limitation of potential damage which can be caused by AEs. Predicting characteristics of AEs is indispensable for assessing this damage. Characteristics of a blast wave generated by an

${ }^{1}$ List of abbreviations used in this paper is given in Appendix.
AE can be predicted either by experiment (direct measuring) or by means of mathematical modelling. Full-scale and scaled experiments imitating AE and allowing a direct measurement of blast wave characteristics can be expensive and cover only a part of values of the random factors which determine these characteristics (e.g. [4]).

In some cases, predicting effects of AEs is possible by combining mathematical modelling and data from direct measurements of AEs [5, 6]. However, the available knowledge can sometimes allow predicting accidental actions merely by mathematical modelling. It can be carried out by applying a stochastic (Monte Carlo) simulation of accidents inducing accidental actions [7]. Such modelling is fully applicable to accidents involving an AE on the road.

The stochastic simulation allows coping with considerable uncertainties related to AEs. Formally, the simulation can be used for propagating these uncertainties through mathematical models of AEs. The uncertainties themselves can be expressed by means of a quantitative risk assessment (QRA). QRA is well suited to dealing with sparseness of statistical data on man-made accidents and so accidents involving AEs on the road $[8,9]$. 
This paper proposes an approach to a simulationbased forecasting effects of an AE which may take place on the road. The result of this forecasting is a probabilistic model describing possible effects which can be induced by this AE. The model can be applied to assessing potential damage to exposed structures in the framework of QRA or, alternatively, it can be used for specifying traditional, deterministic intensities of the effects for the conventional structural analysis.

The paper consists of two parts. The first part develops a procedure for simulating the accident involving $\mathrm{AE}$ on the road and selecting the probabilistic model suitable to describe mechanical effects of this AE. The second part presents a case study which illustrates an application of the proposed procedure [10].

\section{Looking at the road accident from the standpoint of quantitative risk analysis}

AEs and similar dangerous phenomena occurring on the road are, by their very nature, a matter for QRA [11-13]. Mathematical tools of QRA allow dealing with accidents involving AEs in a formalised way. However, many of the QRAs, which consider AEs occurring in transportation, do not approach accidents of this type from the standpoint of structural engineering. The following examination seeks to stress the structural aspect. What is more, the author of the present paper strongly believes that dealing with AEs on the road by the traditional, deterministic means prevailing in the structural engineering does not hold much promise.

Logical models of QRA can be applied to trace causes and consequences of an accident involving AE. The causes of AE can vary substantially. Therefore this paper will be focussed on an accident which can be initiated by a collision of two vehicles. One of them carries explosive substances. Thus AE can be a consequence of vehicular impact. Fig 1 shows an event tree developed for the initiating event $E_{0}=$ "collision of vehicles". Branches of this tree diagram end with the consequences $C_{1}$ to $C_{4}$. The first two, $C_{1}$ and $C_{2}$, are determined mainly by AE.

The standard result of QRA applied to the event tree shown in Fig 1 is the so-called risk profile $\left\{\left(\operatorname{Fr}\left(C_{k}\right)\right.\right.$, $\left.\left.S_{k}\right), k=1,2,3,4\right\}$, where $\operatorname{Fr}\left(C_{k}\right)$ and $S_{k}$ is the frequency and significance of $C_{k}$, respectively (e.g. $\left.[8,9]\right)$. The significances of $C_{1}$ and $C_{2}$ are determined in essence by characteristics of AE. $\operatorname{Fr}\left(C_{1}\right)$ and $\operatorname{Fr}\left(C_{2}\right)$ coincide with the frequency of $\mathrm{AE}$ related to respective event tree branch. This frequency will be denoted next by $\operatorname{Fr}(A E)$, where $A E$ is the random event (re) of $\mathrm{AE}$.

Potential damage to structures from a particular $\mathrm{AE}$ and, more generally, the significance $S_{1}$ and $S_{2}$ can be assessed by means of a probabilistic model describing characteristics of this AE (probabilistic action model, pam). When selected pam can serve as a tool for predicting characteristics of $\mathrm{AE}$ and estimating probabilities of specific damage to structures.

The problem considered in this paper is how to select pam $\operatorname{Fr}(m)$ which expresses the frequency (annual probability) of exceeding the magnitude $m$ of a mechanical effect generated by AE. This model can be schematically defined as $\operatorname{Fr}(A E) \times P(m \mid A E)$, where $P(m \mid A E)$ is the conditional probability of exceeding $m$ given $A E$. In the interests of brevity the selection of $\operatorname{Fr}(m)$ will be considered only for one type of accident, namely, the accident represented by the sequence of re's $E_{0}, E_{1}$, and $E_{2}$ (Fig 1$)$.

The pam $\operatorname{Fr}(m)$ breaks down the modelling problem into smaller problems of estimating the likelihood of $A E$ and modelling characteristics of $\mathrm{AE}$. A more precise definition of the pam $\operatorname{Fr}(m)$ and thus peculiarities of its selection depend on an interpretation of the frequency $\operatorname{Fr}(A E)$ and probability $P(m \mid A E)$. Estimating $\operatorname{Fr}(A E)$ and fitting $P(m \mid A E)$ solely on the basis of the data gained from occurrences of an $A E$ will more often than not be impossible. Data on AEs are usually sparse or unsuitable to a particular situation of exposure to $\mathrm{AE}$, or, what is not uncommon, unavailable at all.

The situation of sparseness or absence of data can be alleviated by mixing hard data (relevant experience data) with subjective information (expert opinions and judgements of analysts) as it is done in QRA [14]. A methodological framework for the combination of hard data and subjective information is provided by a general approach to QRA known as the classical Bayesian approach (CBA) [14, 15]. Principles of CBA will be followed in this paper. In view of the selection of $\operatorname{Fr}(m)$,

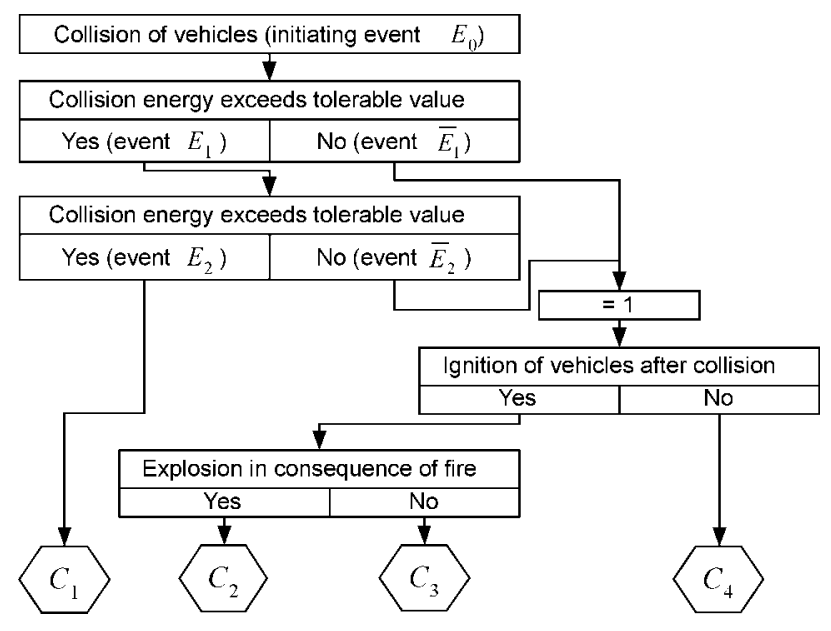

Fig 1. Event tree diagram with the initiating event "collision of vehicles" and tracing the consequences involving an accidental explosion on the road 
CBA may be defined as a tool for estimating $\operatorname{Fr}(A E)$ and $P(m \mid A E)$.

\section{Mathematical model used to predict mechanical effects of explosion on the road}

In line with $\mathrm{CBA}$, the frequency $\operatorname{Fr}(A E)$ and probability $P(m \mid A E)$ are treated as true, even if unobservable and unknown, quantities. Both $\operatorname{Fr}(A E)$ and $P(m \mid A E)$ express the aleatory (stochastic) uncertainty in the re's $A E$ and "exceedance of $m$ given $A E$ ". Another type of uncertainty distinguished in CBA is the epistemic (state-of-knowledge) uncertainty in the true values of $\operatorname{Fr}(A E)$ and $P(m \mid A E)$. Thus a pam based on CBA should incorporate both types of uncertainty.

In terms of CBA, a model of an accidental action can be expressed as a family of probability distributions (pd's) quantifying both aleatory and epistemic uncertainty [7]. As applied to AE, CBA yields the following form of the pam $\operatorname{Fr}(m)$ :

$$
F r_{\mathrm{X}}(\mathrm{x})=\left\{F_{P_{a}}\left(p_{a} \mid \theta_{\alpha}\right),\left(\left(1-F_{\mathrm{X}_{i}}\left(\mathrm{x} \mid \theta_{x i}\right)\right), p_{i}\right), i=1,2, \ldots, n\right\},(1)
$$

where $\boldsymbol{x}$ is the vector of action characteristics; $\boldsymbol{X}$ is the random vector which models an aleatory uncertainty in $\boldsymbol{x} ; p_{a}$ is the frequency of $A E ; P_{a}$ is the random variable (rv) which expresses an epistemic uncertainty in $p_{a}$; $F_{P_{a}}\left(p_{a} \mid \theta_{\alpha}\right)$ is the cumulative distribution function (cdf) of $P_{a}$ with a parameter vector $\boldsymbol{\theta}_{a} ; F_{X_{i}}\left(\boldsymbol{x} \mid \boldsymbol{\theta}_{x i}\right) \quad(i=$ $1,2, \ldots, n)$ are the cdf's of the vector $\boldsymbol{X}$ with the same argument vector $\boldsymbol{x}$ and individual parameter vectors $\boldsymbol{\theta}_{x i}$; $p_{i}$ are the probabilities expressing epistemic uncertainties in cdf's $F_{X_{i}}\left(\boldsymbol{x} \mid \boldsymbol{\theta}_{x i}\right)$. A simplified example of such a pam is the family of hazard curves of ground motion acceleration used in a seismic risk assessment [16].

The definition (1) follows the principles of CBA which require keeping apart the aleatory and epistemic uncertainties in the final form of risk analysis [15]. It follows from the definition (1) that a selection of the pam $F_{r_{X}}(\boldsymbol{x})$ amounts to a selection of cdf $F_{P_{a}}\left(p_{a} \mid \boldsymbol{\theta}_{\alpha}\right)$ and the family of cdf's $F_{\boldsymbol{X}_{i}}\left(\boldsymbol{x} \mid \boldsymbol{\theta}_{x i}\right)$ as well as assignment of the respective weights $p_{i}$.

\section{Selecting the model of accidental explosion on the road}

\subsection{How to deal with virtual lack of direct data on explosion effects?}

In most cases the analyst will face a complete lack of direct data allowing a direct fitting the cdf's $F_{P_{a}}\left(p_{a} \mid \boldsymbol{\theta}_{\alpha}\right)$ and $F_{\boldsymbol{X}_{i}}\left(\boldsymbol{x} \mid \boldsymbol{\theta}_{x_{i}}\right)$. In principle, some data are almost always available, say, from back (postmortem) investigations of one or more analogous accidents. However, it is usually impossible to group the data in samples which are large enough for fitting these cdf's.

The situation of the virtual lack of direct data will be usually encountered when the analyst has to do with unique situations of exposure to AE. However, this situation can not be considered hopeless if the analyst can employ probabilistic and deterministic models describing physical phenomena behind the events $E_{0}$, $E_{1}$, and $E_{2}$. The selection of cdf's $F_{P_{a}}\left(p_{a} \mid \theta_{\alpha}\right)$ and $F_{X_{i}}\left(\boldsymbol{x} \mid \boldsymbol{\theta}_{x_{i}}\right)$ can be based on these models.

Models describing $E_{0}, E_{1}$, and $E_{2}$ are normally developed independently of each other. Therefore it is hardly probable that the entire set of them will be present in a ready-to-use form. In author's experience, extra effort will usually be required to make these models "compatible" with each other.

The central idea of this paper is that in some cases cdf's $F_{P_{a}}\left(p_{a} \mid \boldsymbol{\theta}_{\alpha}\right)$ and $F_{\boldsymbol{X}_{i}}\left(\boldsymbol{x} \mid \boldsymbol{\theta}_{x_{i}}\right)$ can be selected using a stochastic simulation of accident courses (scenarios) represented by $E_{0}, E_{1}$, and $E_{2}$. This accident simulation can generate samples of action characteristics and frequencies of $A E$. The cdf's $F_{\boldsymbol{X}_{i}}\left(\boldsymbol{x} \mid \boldsymbol{\theta}_{x_{i}}\right)$ and $F_{P_{a}}\left(p_{a} \mid \boldsymbol{\theta}_{\alpha}\right)$ can be fitted to the generated samples. The accident simulation can be used for an uncertainty propagation and relate probabilistic models representing $E_{0}, E_{1}$, and $E_{2}$ to cdf's $F_{P_{a}}\left(p_{a} \mid \theta_{\alpha}\right)$ and $F_{X_{i}}\left(\boldsymbol{x} \mid \boldsymbol{\theta}_{x_{i}}\right)$.

\subsection{Procedure for simulating the accident} involving an explosion on the road

The uncertainty propagation via a stochastic simulation of the accident represented by $E_{0}, E_{1}$, and $E_{2}$ is visualised by Fig 2 . It shows the event tree path containing two branching points. A nested loop simulation procedure can be applied to the propagation $[7,17]$. An application of the two loops stems from the necessity to distinguish between the aleatory and epistemic uncertainties. They can be expressed by the model sets $\boldsymbol{\Xi}_{1}:=\left\{F_{P_{0}}\left(p_{0} \mid \boldsymbol{\theta}_{P_{0}}\right), F_{0}\left(z_{0} \mid \boldsymbol{\theta}_{0}\right), F_{\Theta_{0}}\left(\boldsymbol{\theta}_{0}\right)\right\}$ and $\boldsymbol{\Xi}_{2}:=\left\{\boldsymbol{m}_{k}\left(\boldsymbol{z}_{k} \mid \boldsymbol{\theta}_{k}\right), F_{\Theta_{k}}\left(\boldsymbol{\theta}_{k}\right), k=1,2,3\right\}$.

Elements of $\Xi_{1}$ describe the initiating event $E_{0}$ (Fig 1): $F_{P_{0}}\left(p_{0} \mid \boldsymbol{\theta}_{P_{0}}\right)$ is the cdf of the rv $P_{0}$ used to model epistemic uncertainty in the frequency (annual probability) $p_{0}$ of $E_{0}$ (year ${ }^{-1}$, say); $F_{0}\left(\boldsymbol{z}_{0} \mid \boldsymbol{\theta}_{0}\right)$ is the cdf of the random vector $\boldsymbol{Z}_{0}$ used to express aleatory uncertainty in characteristics of $E_{0} ; F_{\Theta_{0}}\left(\boldsymbol{\theta}_{0}\right)$ is the cdf of the random vector $\Theta_{0}$ used to quantify epistemic uncertainty in $\boldsymbol{\theta}_{0} ; \boldsymbol{\theta}_{P_{0}}$ and $\boldsymbol{\theta}_{0}$ are the parameter vectors of $F_{P_{0}}(\cdot)$ and $F_{0}(\cdot)$, respectively.

The model set $\Xi_{2}$ is related to the events succeeding the initiating event, $E_{k}(k=1,2)$ (Fig 1). The models $\boldsymbol{m}_{k}\left(z_{\boldsymbol{k}} \mid \boldsymbol{\theta}_{k}\right)$ describe physical phenomenon 
represented by $E_{k} \mathrm{~s}$, where $z_{k}$ is the input vector and $\boldsymbol{\theta}_{k}$ is the vector of model parameters.

The cdf's $F_{P_{0}}\left(p_{0} \mid \boldsymbol{\theta}_{P_{0}}\right)$ and $F_{\Theta_{k}}\left(\boldsymbol{\theta}_{k}\right) \quad(k=0,1,2)$ in $\Xi_{1}$ and $\Xi_{2}$ can represent prior or posterior pd's depending on the availability of hard data. The role of the models $\boldsymbol{m}_{k}\left(\boldsymbol{z}_{\boldsymbol{k}} \mid \boldsymbol{\theta}_{k}\right)$ in the accident simulation is twofold: (i) output components of $\boldsymbol{m}_{k}\left(\boldsymbol{z}_{\boldsymbol{k}} \mid \boldsymbol{\theta}_{k}\right)$ $(k<3)$ serve as input values of the subsequent model; $\boldsymbol{m}_{3}\left(z_{3} \mid \boldsymbol{\theta}_{3}\right)$ yields action characteristics (Fig 2); (ii) models $\boldsymbol{m}_{1}\left(\boldsymbol{z}_{1} \mid \boldsymbol{\theta}_{1}\right)$ and $\boldsymbol{m}_{2}\left(\boldsymbol{z}_{2} \mid \boldsymbol{\theta}_{2}\right)$ are also used to decide which of the alternative accident courses represented by the event tree branching takes place in the $j$ th repetition of the nested loop (Fig 2).

The rule used to decide at the two branching points represented by the complementary events $E_{k}$ and $\bar{E}_{k}$ $(k=1,2)$ can be expressed as follows:

$$
\mathbf{1}\left(z_{k j} \mid \theta_{k l}\right)= \begin{cases}1 & \text { if } \boldsymbol{m}_{k}\left(z_{k j} \mid \theta_{k l}\right) \in \omega_{k} \text { (event } E_{k} \text { occurs), } \\ 0 & \text { if } \boldsymbol{m}_{k}\left(z_{k j} \mid \theta_{k l}\right) \notin \omega_{k} \text { (event } \bar{E}_{k} \text { occurs), }\end{cases}
$$

where $\omega_{k}$ is the output domain defining an occurrence of $E_{k} ; \boldsymbol{m}_{k}\left(z_{k j} \mid \boldsymbol{\theta}_{k l}\right)$ is the model output computed in the $j$ th repetition of the nested loop and the $l$ th repetition of the outer loop.

The $j$ th repetition of the nested loop is terminated and a new one started as soon as the current binary function $\mathbf{1}\left(\boldsymbol{z}_{k j} \mid \boldsymbol{\theta}_{k l}\right)$ takes zero value (Fig 2). The result $\prod_{k=1}^{2} \mathbf{1}\left(\boldsymbol{z}_{k j} \mid \boldsymbol{\theta}_{k l}\right)=1$ means that $A E$ takes place in the $j$ th and $l$ th repetition of the nested and outer loop, respectively. The $l$ th run of the nested loop with $n_{a l}$ occurrences of AE yields a simulated sample of action characteristics, $\boldsymbol{x}_{1 l}, \boldsymbol{x}_{2 l}, \ldots, \boldsymbol{x}_{n_{a l} l}$, and a relative frequency of $A E$ given by $n_{a l} / n_{0}\left(n_{a l} \leq n_{0}\right)$.

The outer loop is repeated $n_{p}$ times and used for sampling the parameter values $p_{0 l}$ and $\boldsymbol{\theta}_{k l}$ from cdf's $F_{P_{0}}\left(p_{0} \mid \boldsymbol{\theta}_{P_{0}}\right)$ and $F_{\Theta_{k}}\left(\boldsymbol{\theta}_{k}\right) \quad(k=0,1, \ldots, 3)$. On sampling $p_{0 l}$ and $\boldsymbol{\theta}_{k l}$, they serve as input of the nested loop. An application of the nested loop procedure requires simulating the accident $n_{p} \times n_{0}$ times. It yields a sample of $n_{p}$ estimates of the frequency $p_{a}$, namely, $f r_{l}=p_{0 l} n_{a l} / n_{0}$ and $n_{p}$ samples of action characteristics $\mathbf{x}_{l}$. Each $\mathbf{x}_{l}$ is defined as $\mathbf{x}_{l}=\left(\boldsymbol{x}_{j l}, j=1,2, \ldots, n_{a l}\right)$. These samples can serve for fitting cdf's $F_{P_{a}}\left(p_{a} \mid \boldsymbol{\theta}_{\alpha}\right)$ and $F_{\boldsymbol{X}_{i}}\left(\boldsymbol{x} \mid \boldsymbol{\theta}_{x i}\right)$ as well as assigning the weights $p_{i}$.

\section{Expert judgement in forecasting effects of accidental explosions on the road}

A need for expert judgement arises from the fact that AEs are typically rare events backed by sparce hard data. The pam $F_{X}(\boldsymbol{x})$ defined by (1) reflects uncertainties expressed by the sets of "lower-level" models $\Xi_{1}$ and $\Xi_{2}$. Given these sets, the selection of $F_{X}(\boldsymbol{x})$ amounts to uncertainty propagation by means of the above simulation-based procedure.

According to the model classification used in QRA, the models $\boldsymbol{m}_{k}\left(\boldsymbol{z}_{\boldsymbol{k}} \mid \boldsymbol{\theta}_{k}\right)$ can be considered to be process

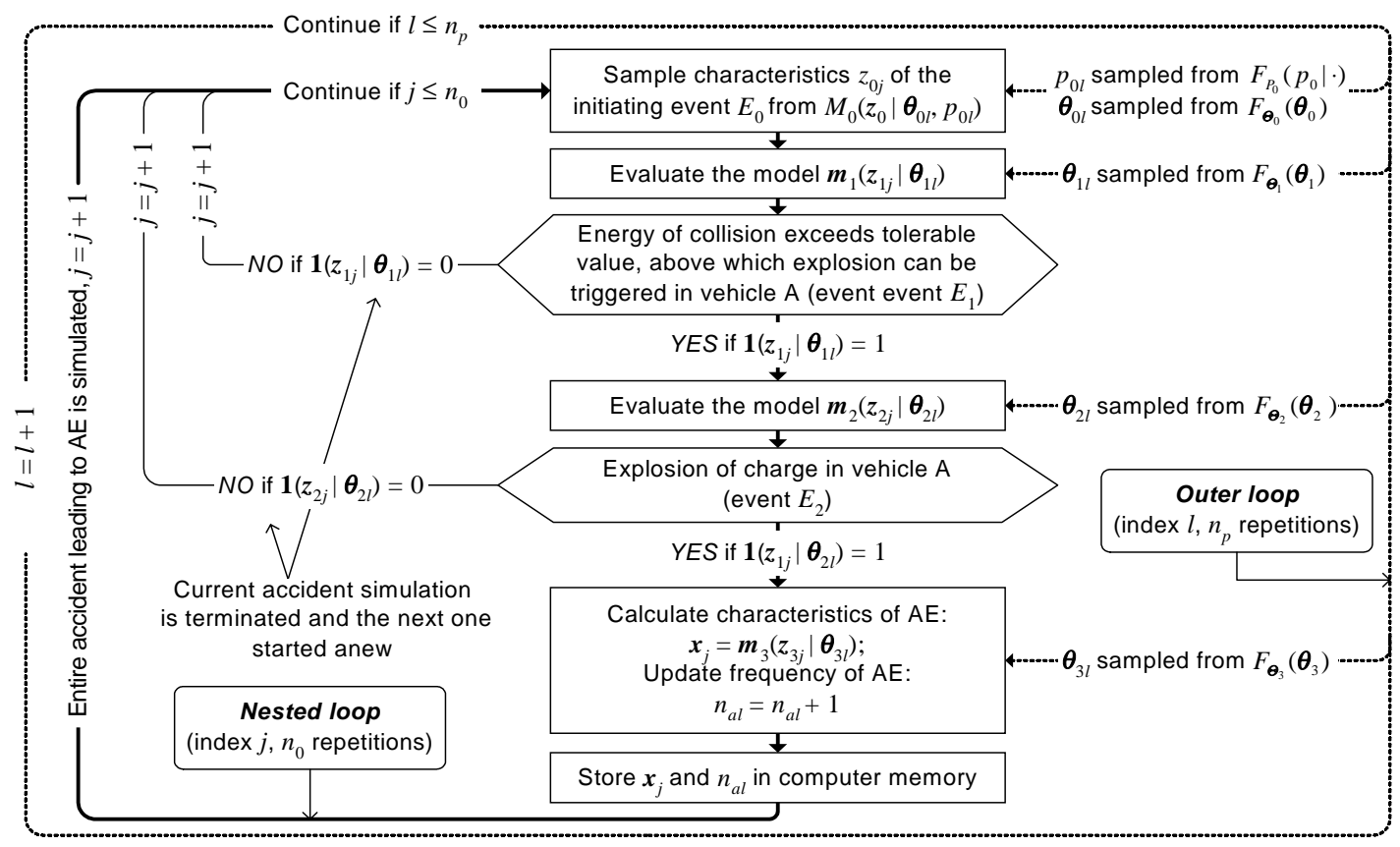

Fig 2. Nested loop procedure used for simulation of the accident involving an accidental explosion on the road 
models, the frequency $p_{0}$ and cdf's $F_{P_{0}}\left(p_{0} \mid \boldsymbol{\theta}_{P_{0}}\right)$, $F_{0}\left(z_{0} \mid \boldsymbol{\theta}_{0}\right)$, and $F_{\Theta_{k}}\left(\boldsymbol{\theta}_{k}\right)$ belong to stochastic models $[14,18]$. The structure of $\Xi_{1}$ and $\Xi_{2}$ depends mainly on the available knowledge about the physical phenomena behind the re's $E_{0}, E_{1}$, and $E_{2}$. A part of this knowledge should be the process models $\boldsymbol{m}_{k}\left(\boldsymbol{z}_{\boldsymbol{k}} \mid \boldsymbol{\theta}_{k}\right)$ which are a prerequisite for an application of the stochastic accident simulation. Another part of the knowledge should be information which allows assigning and updating the stochastic models included in $\Xi_{1}$ and $\Xi_{2}$. This information may consist of available hard data and expert judgements. In some instances expert judgements may be the only source of information for specifying elements of $\Xi_{1}$ and $\Xi_{2}$.

Expert judgement can be incorporated in modelling AEs by applying organisational and mathematical methods developed in the field of QRA. These methods determine choice of experts, formal elicitation of expert opinions as well as quantification and combination of these opinions [14, 18].

Formal means applied to QRA in combining expert judgement with available hard data are provided by the Bayesian statistical theory $[8,18]$. In CBA, elements of this theory are used for updating expert opinions expressed as prior (posterior) distributions of epistemic uncertainty when new information becomes available. These distributions are related to input parameters of such QRA models as event trees and fault trees. Bayesian updating schemes can be used for combining probabilities and p.d.s subjectively assessed by experts [19].

A number of methods for constructing prior distributions have been proposed in the Bayesian statistical theory (see e.g. [20, 21]). Certain of them can be used for constructing prior distributions $F_{P_{0}}\left(p_{0} \mid \theta_{P_{0}}\right)$ and $F_{\Theta_{k}}\left(\boldsymbol{\theta}_{k}\right)$ in situations where relevant hard data are sparse or where such data are not available at all. In the latter case, constructing $F_{P_{0}}\left(p_{0} \mid \boldsymbol{\theta}_{P_{0}}\right)$ and $F_{\Theta_{k}}\left(\boldsymbol{\theta}_{k}\right)$ has to be based on other forms of information. Formal methods for eliciting probabilities and p.d.s from experts have been extensively developed in the field of QRA [19, 22, 23]. Many practical situations covered by the above-mentioned methods can be encountered when simulating accidents involving AEs on the road. These methods can be applied to specifying the epistemic uncertainty measures included in the sets $\Xi_{1}$ and $\Xi_{2}$.

Incorporation of expert judgement in modelling physical phenomena, which can escalate into $\mathrm{AE}$, is to a large degree a problem that can be solved by standard means of QRA. They were developed for a model uncertainty analysis and are closely linked to an expert opinion use [24]. The meaning of model uncertainty and the role of expert judgement in quantifying this uncertainty under the CBA format are discussed in [25].

The practical application of the simulation-based procedure proposed in Sec. 4.2 can be complicated by the fact that the process models $\boldsymbol{m}_{k}\left(\boldsymbol{z}_{\boldsymbol{k}} \mid \boldsymbol{\theta}_{k}\right)$, which embody the state-of-knowledge about the physical phenomena related to the events $E_{1}, E_{2}$, and $A E$, may be deterministic and imperfect (not error free). This can require supplementing deterministic constituents of $\boldsymbol{m}_{k}\left(z_{k} \mid \boldsymbol{\theta}_{k}\right)$ with subjective uncertainty distributions which could allow applying these models in the Bayesian framework. A candidate approach to such a probabilistic "upgrading" of the models $\boldsymbol{m}_{k}\left(\boldsymbol{z}_{\boldsymbol{k}} \mid \boldsymbol{\theta}_{k}\right)$ is the method of adjustment factors [26]. The application of this method to the model $\boldsymbol{m}_{3}\left(\boldsymbol{z}_{3} \mid \boldsymbol{\theta}_{3}\right)$ will be illustrated in the second part of the present paper [10].

Specifying the stochastic models belonging to $\Xi_{1}$ and $\Xi_{2}$ may be a highly case-specific task. It is determined mainly by available knowledge on $E_{k} \mathrm{~s}$ (general information on the physical phenomena related to $E_{k} \mathrm{~s}$, relevant hard data on $E_{k} \mathrm{~s}$, and expert judgements). Therefore, the case study described in the second part of the paper gives methodological ("howto-do" and "where-to-find") descriptions of the procedures which can be applied to assigning and updating elements of $\Xi_{1}$ and $\Xi_{2}$ which are used to quantify expert judgement [10].

\section{Conclusions}

This paper proposed a computational procedure intended for selecting mathematical models for actions induced during accidental explosions (AEs) on the road. It was suggested to choose mathematical models for AEs (action models) in the framework of the quantitative risk assessment (QRA). It was shown that methods of QRA allow considering the specific nature of the rare and difficult-to-predict physical phenomena occurring as AEs. The proposed procedure is underpinned by the classical Bayesian approach to QRA. This approach is well suited to quantifying aleatory (stochastic) and epistemic (state-of-knowledge) uncertainties related to actions induced by AEs on the road.

The procedure implements a stochastic simulation of an accident initiated by a vehicular collision. It relates the aleatory and epistemic uncertainties in characteristics of AE to the uncertainties in the physical phenomena, the sequence of which may end up in AE. Thus the procedure serves the purpose of propagating uncertainties. The action model selected by applying the procedure expresses both aleatory and epistemic uncertainty related to AE.

The practical application of the proposed 
procedure requires establishing mathematical models describing the physical phenomena which precede $\mathrm{AE}$. They determine initial information (hard data and expert judgements) which must be collected and transformed via uncertainty propagation into an action model. The simulation of the accident involving $\mathrm{AE}$ is not an end in itself. A preparation of this simulation indicates what initial information is to be gained to allow using the available models. This is an important, albeit indirect result of the analysis of an accident involving AE. Clearly, results of the simulation will be conditioned on the models being applied.

The classical Bayesian approach to QRA was considered in this paper as a means of combining hard data and expert judgements within the problem of predicting AE. This approach allows compensating sparce experience data on AE by subjective information. The number of recipes given in QRA and Bayesian statistical theory for a formal use of subjective information is relatively large, albeit not sufficiently systemised for practical use.

The simulation-based procedure proposed in the paper can be used for design of future structures (assessment of existing structures) which are built in vicinity of roads used for transportation of explosive substances and goods. The second part of the present paper contains a case study which illustrates an application of the proposed procedure [10].

\section{References}

1. Oggero, A. et al. A survey of accidents occurring during the transport of hazardous substances. Journal of Hazardous Materials (Article in Press), 2006.

2. Kletz, T. A. Transportation of hazardous substances: The UK scene. Process Safety Progress, Vol 5, No 3, 2004, p. 160-164.

3. Planas-Cuchi, E. et al. Explosion of road tanker containing liquefied natural gas. Journal of Loss Prevention in Process Industries, Vol 17, No 4, 2004, p. 315-321.

4. Bulson, P. S. Explosive loading on engineering structures. London etc: E \& FN Spon, 1997. 236 p.

5. Vaidogas, E. R. Accidental explosions on the railway: Simulation-based prediction of the damage to nearby buildings. Transport, Vol XX, No 6, 2005, p. 265-273.

6. Vaidogas, E. R. Bayesian bootstrap: Use for estimating probabilities of accidental damage to structures. In: Advances in safety and reliability, Proc ESREL 2005, Ed K. Kołowrocki, Vol 2, Leiden etc: Balkema, 2005, p. 1973-1980.

7. Vaidogas, E. R. Actions imposed on structures during man-made accidents: Prediction via simulation-based uncertainty propagation. Journal of Civil Engineering and Management, Vol XI, No 3, 2005, p. 225-242.
8. Aven, T. Foundations of risk analysis. Chichester etc.: Wiley, 2003. 190 p.

9. Kumamoto, H.; Henley, E. J. Probabilistic risk assessment for engineers and scientists, 2nd ed. New York: IEEE Press, 1996. 511 p.

10. Vaidogas, E. R. Simulation-based forecasting effects of an accidental explosion on the road. Part II: Case study. Transport, Vol XXI, No 4, 2006 (Article in Press).

11. Fabiano, B. et al. Dangerous goods transportation by road: from risk analysis to emergency planning. Journal of Loss Prevention in Process Industries, Vol 18, No 4-6, 2005, p. 403-413.

12. Bubbico, R. et al. Risk analysis for road and rail transport of hazardous materials: a simplified approach. Journal of Loss Prevention in Process Industries, Vol 17, No 6, 2004, p. 477-482.

13. Gheorghe, A. V. et al. Comprehensive risk assessment for rail transportation of dangerous goods: a validated platform for decision support. Reliability Engineering \& System Safety, Vol 88, No 3, 2005, p. 247-272.

14. Apeland, S.; Aven, T.; Nilsen, T. Quantifying uncertainty under predictive, epistemic approach to risk analysis. Reliability Engineering \& System Safety, Vol 75, 2002, p. 93-102.

15. Aven, T.; Pörn, K. Expressing and interpreting the results of quantitative risk analyses. Review and discussion. Reliability Engineering \& System Safety, Vol 61, 1998, p. 3-10.

16. Ravindra, M. K. Seismic risk assessment. In: Sundararajan, C., editor. Probabilistic Structural Mechanics Handbook. New York: Van Nostrand, Reinhold, 1993, p. 429-464.

17. Hofer, E.; Kloss, M. et al. An approximate epistemic uncertainty analysis approach in the presence of epistemic and aleatory uncertainties. Reliability Engineering \& System Safety, Vol 77, 2002, p. 229-238.

18. Kaplan, S.; Burmaster, D. How, when, why to use all the evidence. Risk Analysis, Vol 19, 1999, p. 55-62.

19. Clement, R. T.; Winkler, R. L. Combining probability distributions from experts in risk analysis. Risk Analysis, Vol 19, 1999, p. 187-202.

20. Smith, A. F. M. An overview of the Bayesian approach. In: Sander, P.; Badaux, R. editors. Bayesian Methods in Reliability. Dordrecht etc.: Kluwer, 1991, p. 15-79.

21. Siu, N. O.; Kelly, D. Bayesian parameter estimation in probabilistic risk assessment. Reliability Engineering \& System Safety, Vol 62, 1998, p. 89-116.

22. Mosleh, A.; Bier, V. M.; Apostolakis, G. A critique of current practice for the use of expert judgement in probabilistic risk assessment. Reliability Engineering \& System Safety, Vol 20, 1988, p. 63-85.

23. French, S.; Cooke, R. M.; Wiper, M. P. The use of expert judgement in risk assessment. In: Sander, P.; Badaux, R., editors. Bayesian methods in reliability. Dordrecht et al.: Kluwer, 1991, p. 119-134.

24. USNRC. An approach for using probabilistic risk assessment in risk decisions on plant-specific changes 
to licensing basis. Regulatory Guide 1.174. USNRC Draft DG-1061, 1997.

25. Nilsen, T.; Aven, T. Models and model uncertainty in the context of risk analysis. Reliability Engineering \& System Safety, Vol 79, 2003, p. 309-317.

26. Zio, E.; Apostolakis, G. E. Two methods for the structured assessment of model uncertainty by experts in performance assessment of radioactive waste repositories. Reliability Engineering \& System Safety, Vol 54, 1996, p. 225-241.

\section{Appendix. Abbreviations used in the paper}

$\begin{array}{lll}\mathrm{AE} & = & \text { accidental explosion } \\ \mathrm{CBA} & = & \begin{array}{l}\text { classical Bayesian } \\ \text { approach }\end{array} \\ \mathrm{cdf}(\mathrm{cdf} \text { 's) } & = & \begin{array}{l}\text { cumulative distribution } \\ \text { function(s) }\end{array} \\ \text { pam } & = & \text { probabilistic action model } \\ \text { pd (pd's) } & = & \begin{array}{l}\text { probability } \\ \text { distributions(s) }\end{array} \\ \mathrm{QRA} & = & \begin{array}{l}\text { quantitative risk } \\ \text { assessment }\end{array} \\ \text { re (re's) } & = & \text { random event(s) } \\ \text { rv (rv's) } & = & \text { random variable(s) }\end{array}$

